\section{Correlated Functional Changes of the Prefrontal Cortex in Twins Induced by Classroom Education of Second Language}

Kuniyoshi L. Sakai ${ }^{1,2}$, Kunihiko Miura ${ }^{2,3}$, Nobuko Narafu ${ }^{2,3}$ and Yukimasa Muraishi ${ }^{2,3}$

${ }^{1}$ Department of Cognitive and Behavioral Science, Graduate School of Arts and Sciences, University of Tokyo, Komaba, 38-1 Komaba, Meguro-ku, Tokyo, Japan, ${ }^{2}$ CREST, Japan Science and Technology Agency, Kawaguchi-shi, Japan and ${ }^{3}$ The Secondary Education School Attached to the Faculty of Education of the University of Tokyo, 1-15-1 Minamidai, Nakano-ku, Tokyo, Japan

studying English for the first time at a secondary education school in Japan. We targeted twins as subjects, because it is intriguing to ask whether shared factors of twins actually influence their language abilities and neural substrates for Japanese (L1) and English (L2). For 2 months, the students participated in intensive training in English verbs as part of their standard classroom education (see Materials and Methods). All first-year students $(n=117)$ in three classes received the training and in no case did both members of a twin pair belong to the same class. To evaluate directly the brain's changes in activation due to this training, the twins completed two sets of functional magnetic resonance imaging (fMRI) sessions, one before the training (day 1) and one after the training (day 2). Figure 1 illustrates the experimental paradigm with four tasks used in fMRI sessions: an English verb-matching (EM) task, an English past tense (EP) task, a Japanese verb-matching (JM) task and a Japanese past tense (JP) task. There were two EP task blocks in a single fMRI session: one block (EPr) with seven regular verbs and one irregular verb (at a randomized position) and the other (EPi) with seven irregular verbs and one regular verb. General cognitive factors such as word recognition and response selection were controlled by the EM and JM tasks, which were directly compared with the EP and JP tasks, respectively. A particular challenge in this study was to assess the effect of an educational method used in classroom lessons directly in terms of brain activation.

\section{Materials and Methods}

\section{Participants}

Participants in the present fMRI study were 18 native Japanese speakers (11 females and 7 males, all aged 13) without any neurological problems. They consisted of seven monozygotic and two dizygotic twin pairs, as determined by serological analyses of blood types and DNA markers. All subjects showed right-handedness (laterality quotients: 44-100) according to the Edinburgh inventory. One dizygotic twin pair had lived in the United States from age 7-9 with English exposure and thus the data on this pair were excluded from the analyses. None of the other pairs had learned English past tense verbs before the present training. The participants were in a supine position in the magnet, while the participant's head was immobilized with padding inside the radio-frequency coil. The data from one monozygotic twin pair were not included in the analyses because of head movements during scanning. The current results thus based on seven twin pairs (eight females and six males). Informed consent was obtained from all twin individuals and their parents. This study was approved by the Secondary Education School Attached to the Faculty of Education of the University of Tokyo and by the institutional review board of the University of Tokyo, Komaba.

\section{Tasks}

In each $3 \mathrm{~s}$ trial of the fMRI experiments, word or pseudoword stimuli in yellow letters against a dark background were presented visually at 
A

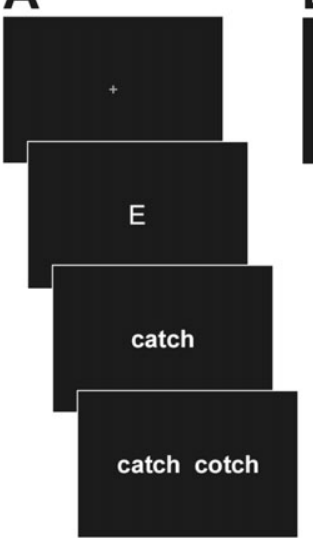

B

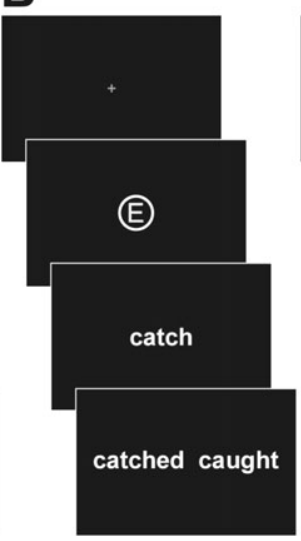

C

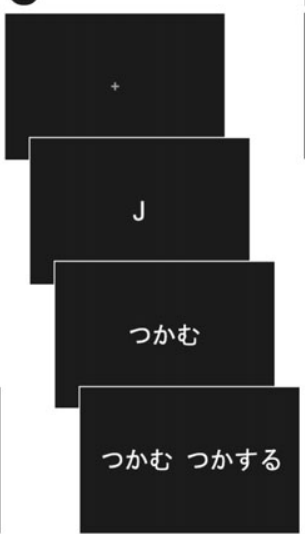

D

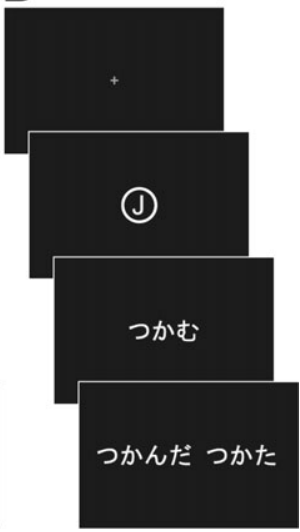

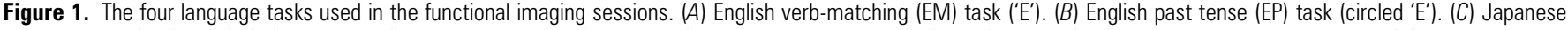

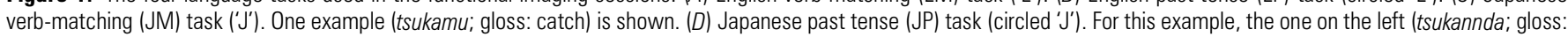

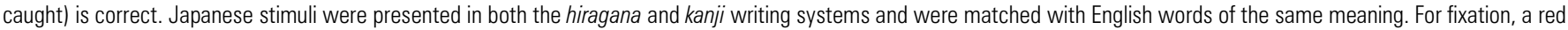
cross was always shown at the center of the screen, but it was omitted from the stimuli. See Materials and Methods for details of each trial.

the center of the screen (Fig. 1). First, a symbol of 'E' (EM), circled ' $E$ ' (EP), 'J' (JM) or circled 'J' (JP) was shown for $400 \mathrm{~ms}$ at the initiation of every trial, indicating the task to be performed. Next, a present tense verb was shown for $1300 \mathrm{~ms}$, during which the subject read it silently for EM and JM, or read its past tense form silently for EP and JP if they knew it (otherwise, they read the presented verb). Finally, two choice stimuli were presented for $1000 \mathrm{~ms}$ and the subjects chose the same present tense verb for EM and JM, or chose the correct past tense form for EP and JP even if it was unfamiliar to them, by pushing one of two buttons. The time limit given for responses was as short as $1000 \mathrm{~ms}$, so that responses without preplanning were discouraged. No feedback on performance was given to any subject.

The four tasks were conducted in a block design and the stimuli used in each block were different. Six trials were tested in each EM block and eight trials were tested in each block for the other tasks. The EM task served as the baseline; one EM block and one block each of JM, EPr, EPi and JP were alternately presented (a total of nine blocks) for each fMRI session. Ten fMRI sessions were carried out in one day. The order of tasks was counterbalanced across subjects. The stimulus presentation and behavioral data collection were controlled by LabVIEW software and interface (National Instruments, Austin, TX). The subjects wore earplugs and an eyeglass-like MRI-compatible display (resolution: $800 \times 600$, VisuaStim XGA; Resonance Technology Inc., Northridge, CA). The same stimuli and tasks were used on days 1 and 2 to equalize any material-dependent conditions except practice effects.

\section{Training}

For each classroom lesson, two bingo sheets (a $5 \times 5$ matrix on each sheet, with rows $B, I, N, G$ and $O$ as well as a 'free' square in the centre) were prepared. The first sheet contained a list of eight verb pairs matching present tense of a verb to the past tense (e.g. close-closed), while the second sheet contained eight past tense verbs only. Before each lesson began, the students were asked to complete these sheets by filling in each row with five of eight verb items, one item per square. During the game with the first sheet, the teacher called out each row's name (circularly in the order of B, I, N, $\mathrm{G}$ and $\mathrm{O}$ ) and any verb pair; each student then marked the square of that row in which the called verb pair had been already written. 'Bingo!' is announced by the student when one row, column, or diagonal line of five squares has been marked. The first game continues until one student announces 'Bingo! Bingo!' when all squares are marked. During the game with the second sheet, the teacher called out each row's name and any present tense verb and each student then marked the square of that row in which the corresponding past tense verb had been already written. The purpose of the second game is to conjugate English verbs from present to past tense. It takes $\sim 5$ min to complete these two games.
A set of 64 verbs with regular inflection and a set of 64 verbs with irregular inflection were prepared and they were grouped on 16 bingo sheets as follows: for class A, either regular or irregular verbs with similar sound patterns (e.g. 'bring-brought' and 'think-thought') were grouped together; for class $\mathrm{B}$, either regular or irregular verbs were grouped, while verbs with different sound patterns were mixed together; and for class $\mathrm{C}$, all verbs were grouped randomly. From these sets, we chose 48 verbs ( 24 regular and 24 irregular) for the fMRI sessions and 50 verbs (25 regular and 25 irregular) for an English past tense examination. Through the 2 month period, each class had 25 lessons, going through the nine bingo sheets twice. The original method of using bingo games in class to teach English words was developed by Mr Katsuhiko Osa.

\section{fMRI Data Acquisition and Analyses}

The fMRI scans were conducted using a $1.5 \mathrm{~T}$ scanner (Stratis II, Premium; Hitachi Medical Corporation, Tokyo, Japan). Using a gradient-echo echo-planar imaging sequence (repetition time, $3 \mathrm{~s}$; echo time, $50.5 \mathrm{~ms}$; acquisition time, $1850 \mathrm{~ms}$; resolution, $3 \times 3 \mathrm{~mm}^{2}$ ), we scanned 16 horizontal slices, each $6 \mathrm{~mm}$ thick and having a $1 \mathrm{~mm}$ gap, covering from $z=-49$ to $62 \mathrm{~mm}$. We performed group analyses using SPM99 statistical parametric mapping software (Wellcome Department of Imaging Neuroscience, London, UK). We realigned the functional volume data in multiple sessions and removed sessions that included data with a translation of $>2 \mathrm{~mm}$ in one of the three directions and a rotation of $>1.4^{\circ}$. The data were normalized to the standard brain, resampled every $3 \mathrm{~mm}$ using bilinear interpolation and smoothed with an isotropic Gaussian kernel of $12 \mathrm{~mm}$ full width at half maximum. Task-specific effects were estimated with a general linear model using a boxcar waveform convolved with the canonical hemodynamic response function. Random effects analyses were performed for all of intersubject comparisons. The statistical parametric maps in each comparison were thresholded at corrected $P<$ 0.05 for the cluster level and uncorrected $P<0.001$ for the voxel level. Statistical significance of positive correlation between the two individuals in each twin pair was tested using Spearman rank correlation (one-sided), which is resistant to outliers, i.e. high leverage points

\section{Results}

By the end of training, the students showed significant improvements in identifying the correct English past tense verbs, as assessed by past tense examination in class. The same examination was administered without consultation before and after the 2 month training period. Given a verb in the present tense (e.g. 'bring'), the students marked which of two possible past tense verb forms (e.g. 'brought' or 'bringed') was 
correct. For present tense verbs that seemed unfamiliar, the students were instructed not to mark either of the two past tense forms. The mean scores of the first and second examination for all 117 students were $14 \pm 3.4 \%$ and $61 \pm 2.9 \%$ (mean \pm SE), respectively. There was no statistically significant difference in examination scores among the three classes $(P>0.1)$.

The seven twin pairs $(n=14)$ also showed better scores for the second examination: regular verbs, $64 \pm 5.7 \%$; and irregular verbs, $55 \pm 6.4 \%$. There was no significant difference between the scores of regular and irregular verbs [paired $t$-test, $t(13)=$ $1.3, P>0.2]$ and thus the score data were collapsed. The two individuals in each pair exhibited marginally correlated performances on the second examination $(r=0.69$, Spearman rank correlation coefficient $r_{\mathrm{S}}=0.62, P=0.06$; Fig. $2 A$ ), in spite of large interpair differences in performance improvements. In order to establish that this correlation within each pair occurred not by chance but due to factors that individual twins had in common with their counterpart twins within a pair, each individual was randomly assigned to a new pair to produce $>100$ permutations. These random pairings resulted in the normal distribution with mean $r=0.0$ and $\mathrm{SD}=0.40$. Therefore, the above correlation coefficient of $r=0.69$ reaches statistical significance at $P<0.05(Z>1.64)$.

The accuracies of the seven twin pairs for EM, JM and JP, performed during fMRI sessions, was 90, 95 and 91\%, respectively,
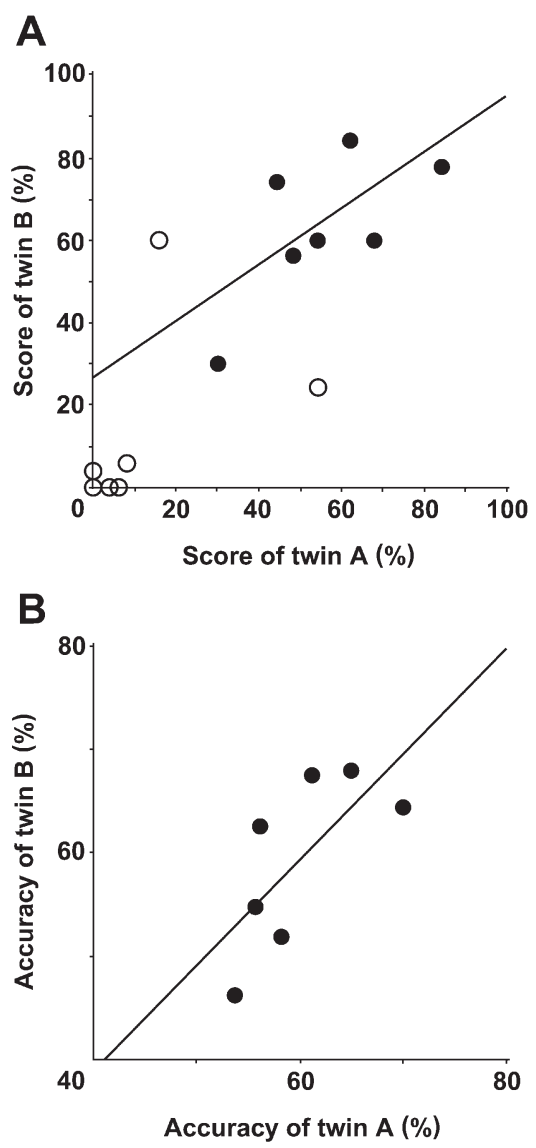

Figure 2. Significant correlation for performances on past tense verbs within each pair of twins. (A) The score of an English past tense examination is plotted for each pair of twins (identified as twin $A$ and twin B). Open and filled dots denote data on day 1 (before training) and day 2 (after training), respectively. $(B)$ The accuracy for the EP tasks in fMRI sessions on day 2 is plotted for each pair of twins. A regression line for day 2 data is shown for each panel. all of which remained unchanged for days 1 and 2. According to an analysis of variance (ANOVA) with two variables [task $(\mathrm{EM}, \mathrm{JM}$ and JP) $\times$ day (days 1 and 2$)$ ], accuracy data showed significant main effects of task $(P<0.0005)$, while the main effects of day and their interactions were not significant $(P>$ 0.05). In the EP tasks, forced two-choice were imposed even if present tense verbs seemed unfamiliar, and the students chose verbs with incorrect regular inflections (e.g. 'selled' rather than 'sold') more often than those with incorrect irregular inflections (e.g. 'smold' rather than 'smelled', in analogy with 'sell'-'sold'). For day 1, the mean accuracies for the past tense forms of regular verbs (seven stimuli in EPr and one in EPi) and that for irregular verbs (seven stimuli in EPi and one in EPr) were $79 \pm 3.9$ and $22 \pm 3.9 \%$, respectively $(51 \pm 1.0 \%$ on average); for day 2 , they were $77 \pm 3.7$ and $43 \pm 6.3 \%$, respectively ( $60 \pm 1.9 \%$ on average). An ANOVA on these accuracy data confirmed the significant interaction of verb type by day $(P<0.05)$. The unusually high accuracy for regular verbs was probably due to mere guesswork of choosing verbs ending in '-ed' when past tense verbs cannot be recalled with confidence. The individuals within the twin pairs were highly correlated with each other in their overall performances of the EP tasks for day $2\left(r=0.71, r_{\mathrm{S}}=0.79, P<0.025\right.$; Fig. $\left.2 B\right)$. The permutation procedure, which resulted in the normal distribution with mean $r=0.0$ and $\mathrm{SD}=0.39$, also confirmed statistical significance at $P<0.05$.

In parallel with the performance improvements for English past tense verbs, we observed clear activation increases in cortical regions (Fig. 3 and Table 1). When EPr blocks were contrasted with EM blocks in a random effects analysis for day 2, major activations were found in the following regions: the dorsal region of the left inferior frontal gyrus (IFG) spanning the opercular part and the triangular part of IFG (F3op/F3t), the left F3t/F2 [Brodmann's area (BA) 46] and the right cerebellum (lobule VI, extending to Crus I; Fig. $3 A$ and Table 1). All of these activations were absent in EPr - EM for day 1. Although the apparent performance for regular verb inflection was $\sim 80 \%$ for both days 1 and $\mathbf{2}$, the almost pure guesswork involved in choosing verbs ending in '-ed' for day 1 did not sufficiently induce this activation. When EPi blocks were contrasted with EM blocks for day 2, significant activations were observed in the following regions: the left dorsal IFG (F3op/F3t), the left ventral IFG spanning the triangular part and the orbital part of IFG (F3t/F3O), the left F3t/F2 (BA 46), the left angular gyrus and supramarginal gyrus (AG/SMG) and the right cerebellum (Fig. $3 B$ and Table 1 ). All of these activations were absent in EPi - EM for day 1. It may be notable that the activated regions of the left IFG for day 2 in EPi - EM were slightly wider than those in EPr - EM (Table 1) and that the left AG/SMG activation was observed only in EPi - EM. However, the direct comparison EPi - EPr showed no significant activation for day 2 .

Given the consistency of activations between the EPr and EPi blocks, we performed a paired $t$-test of day 2 - day 1 as a second level analysis of (EPr + EPi) - EM. This random effects analysis resulted in a single activated region of the left dorsal IFG (Fig. 3C and Table 1). Moreover, the JP - JM contrast for day 2, with the same statistical analyses as used in EPr - EM and $\mathrm{EPi}$ - EM, revealed the left IFG activation that extends from dorsal (F3op/F3t) to ventral (F3t/F3O) regions (Fig. $3 D$ and Table 1). This left IFG activation was stable and remained unchanged between days 1 and 2 , in that a paired $t$-test of 


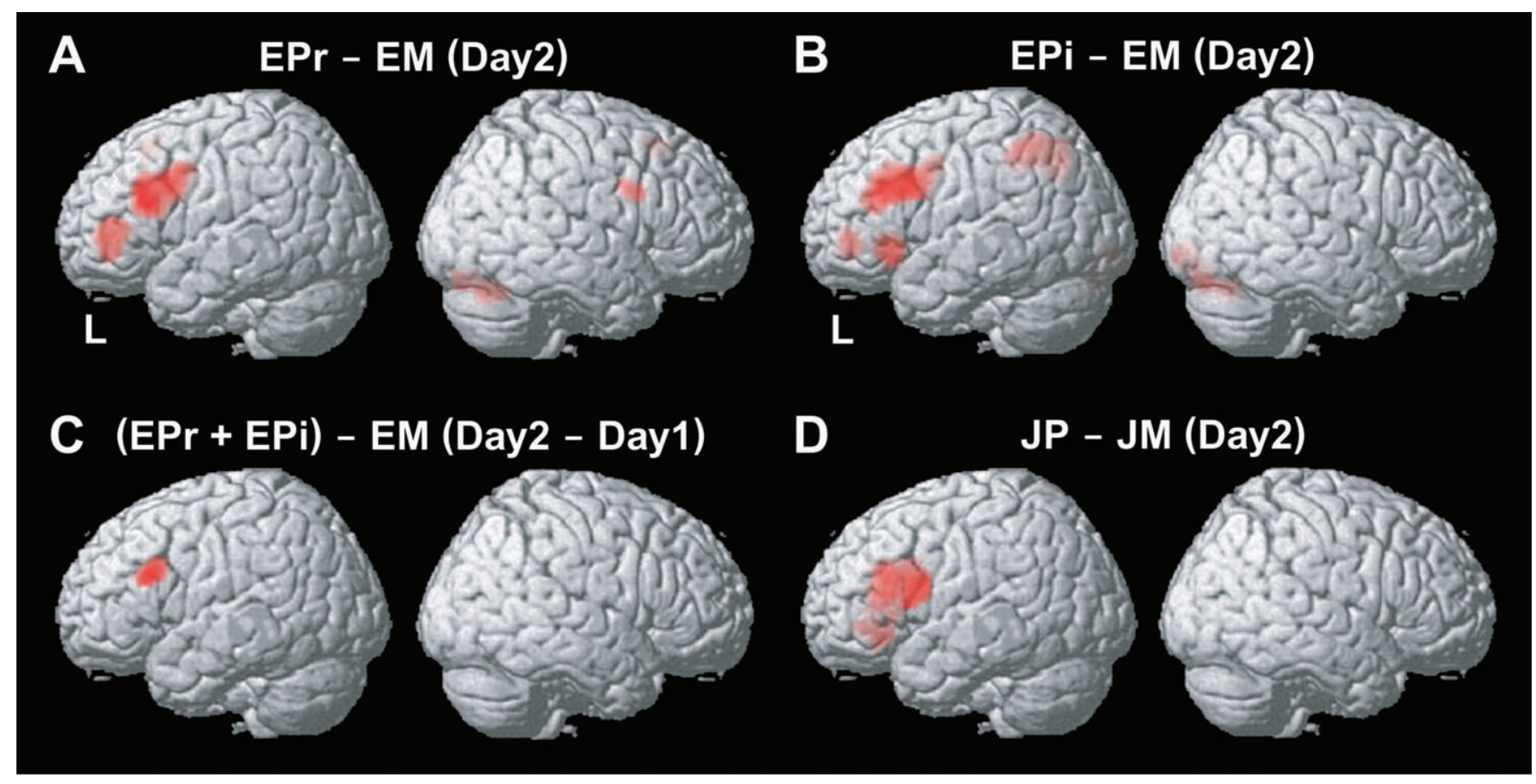

Figure 3. The neural systems for processing L1 and L2 past tense verbs. The activated regions were projected onto a surface-rendered standard brain. $(A) E P r-E M$ for day 2. (B) EPi - EM for day 2. The most prominent activation change was observed in the left (L) inferior frontal gyrus (IFG). (C) A paired $t$-test of day $2-$ day 1 as a second level analysis of

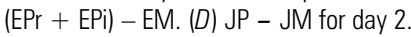

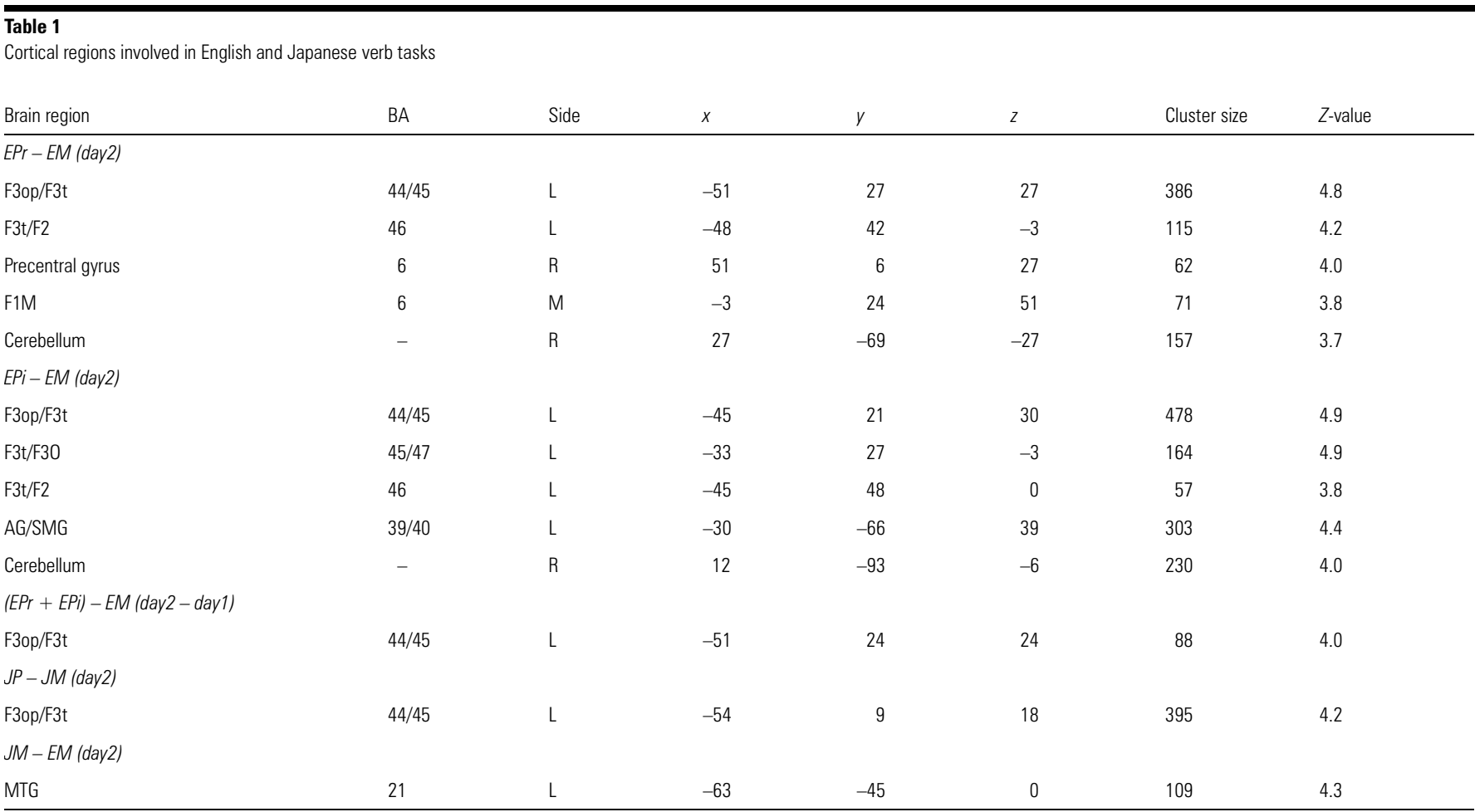

Stereotactic coordinates $(x, y, z)$ in the standard brain space $(\mathrm{mm})$ as defined by Montreal Neurological Institute (MNI) are shown for each local maximum of Z-values in the contrasts indicated. BA,

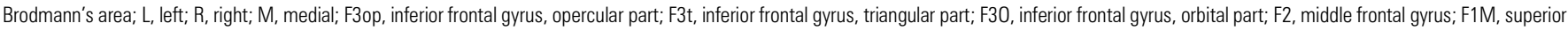
frontal gyrus, medial; $A G / S M G$, angular gyrus and supramarginal gyrus; MTG, middle temporal gyrus.

day 2 - day 1 as a second level analysis of JP - JM showed no significant activation. It is thus striking to note that the left prefrontal regions activated for the English past tense verbs exactly match the regions activated for the Japanese past tense verbs. Indeed, the conjunction analysis of $(\mathrm{EPr}+\mathrm{EPi})-\mathrm{EM}$ and JP - JM revealed significant activation only in the left dorsal IFG 
(cluster size, 56 voxels). These results suggest that the activation increases in the left dorsal IFG represent the acquisition of past tense knowledge.

We next examined whether or not shared factors of twins influence the functional changes observed here. Consistent with the correlated performances for the past tense examination and the EP tasks, the activation increases at one local maximum $(-45,21,30)$ of the left dorsal IFG (F3op/F3t), which were calculated for (EPr + EPi) - EM across days 1 and 2 $(0.21 \pm 0.04 \%$; mean $\pm \mathrm{SE})$, showed a highly significant correlation within each pair of twins $\left(r=0.80 ; r_{\mathrm{S}}=0.79, P<0.025\right)$ (Fig. $4 A$ ). The permutation procedure, which resulted in the normal distribution with mean $r=0.0$ and $\mathrm{SD}=0.39$, also confirmed statistical significance at $P=0.02$. The activation changes of the same region in JP - JM across days 1 and $2(0.05$ $\pm 0.06 \%$, i.e., no significant change) showed no positive correlation within each pair of twins $(r=-0.46)$, indicating that the significant correlation is selective to $\mathrm{L} 2$ acquisition. In contrast, the activation change at one local maximum $(27,-69,-27)$ of the right cerebellum, which were also calculated for $(\mathrm{EPr}+\mathrm{EPi})$ - EM across days 1 and 2, showed no significant correlation $(r=-0.017)$, and the activation was more variable among individuals (Fig. $4 B$ ). It is thus surprising that functional changes specifically observed in the left dorsal IFG are influenced by
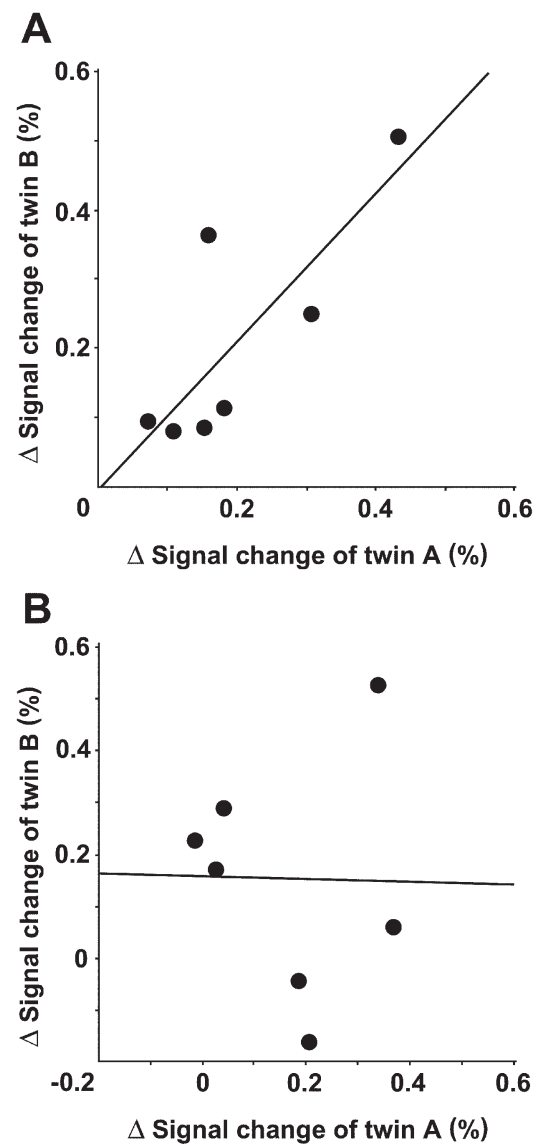

Figure 4. The region-specific correlated activation increases for $L 2$ within each pair of twins. (A) The activation increases ( $\Delta$ signal change: day $2-$ day 1$)$ of the left dorsal IFG $(-45,21,30)$ in $(E P r+E P i)-E M$, plotted for each pair of twins (identified as twin A and twin B). (B) The activation changes of the right cerebellum $(27,-69,-27)$ in (EPr $+\mathrm{EPi})-\mathrm{EM}$ across days 1 and 2. A regression line is shown for each panel. shared factors for each pair of twins in a highly predictive manner.

Finally, we tested whether or not the activation increases in the left dorsal IFG predicted the extent to which each individual subject improved his/her knowledge of the past tense. We found a significant positive correlation $(r=0.63$, Fisher's $r$ to $Z$ transformation $Z=2.5, P<0.02$, two-sided) between the improvements in examination scores and the activation increases in (EPr + EPi) - EM across days 1 and 2 (Fig. 5). Therefore, we conclude that the amount by which activation increases in the left dorsal IFG is a good indicator of individual improvement in acquiring L2 past tense knowledge.

\section{Discussion}

The results are striking in three ways. First, after classroom training in past tense verbs in L2, each 13-year-old twin pair exhibited significantly correlated performances for both the past tense examination and the EP tasks. Secondly, the left dorsal IFG exhibited significantly correlated activation increases within each pair of twins and the increases were positively correlated with individual performance improvements. These results suggest that the functional changes specifically observed in the left dorsal IFG are susceptible to shared genetic and environmental factors for each twin in a surprisingly predictive manner. Thirdly, the cortical plasticity for L2 acquisition was guided toward the L1 specialization of the left dorsal IFG, observed at least at the age of 13 , in spite of notable differences between L1 and L2 in the students' linguistic knowledge and in their performance in making past tense forms. The present fMRI study thus successfully elucidates one type of cerebral mechanisms underlying language acquisition, indicating how new linguistic knowledge is acquired and represented in individual brains. To our knowledge, the present study is the first direct demonstration that classroom education, if properly executed, can change the function of the prefrontal cortex.

The left IFG activation observed for the EP tasks on day 2 and for the JP task on both days cannot be explained by task difficulty or other domain-general factors, because the EP tasks were easier on day 2 than on day 1 and the JP task was much easier for the subjects. We have recently established

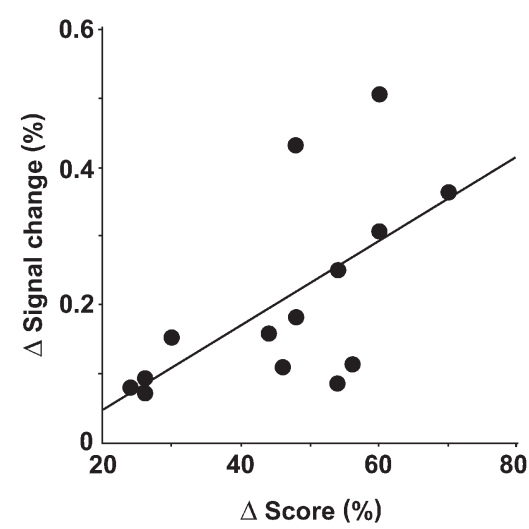

Figure 5. Significant correlation between L2 performance improvements and activation increases in the left dorsal IFG. For all individual participants $(n=14)$, the activation increases ( $\Delta$ signal change: day $2-$ day 1$)$ of the left dorsal IFG $(-45,21,30)$ in $(E P r+E P i)-E M$ are plotted against improvements in examination scores ( $\Delta$ score: day 2-day 1). A regression line is shown. 
that the left IFG is selectively involved in syntactic processes and that its function is indeed separable from domain-general cognitive factors such as task difficulty and short-term memory (Hashimoto and Sakai, 2002; Sakai et al., 2002, 2003; Suzuki and Sakai, 2003). We also excluded the possible involvement of familiarization in discriminating and choosing real words over nonwords. If such a general factor had resulted in the left IFG activation for the EP and JP tasks, the EM and JM tasks would have produced comparable activation, and thus neither $(\mathrm{EPr}+\mathrm{EPi})$ - EM nor JP - JM would have shown any activation. Moreover, the left IFG activation is independent of reading skills and semantic processes associated with presented verbs. The contrast between the two language tasks JM - EM would extract highly developed reading skills and semantic processes of L1, but this contrast resulted in significant activation of the left middle temporal gyrus (MTG) alone (Table 1). Furthermore, the absence of significant activation in a paired $t$-test of day 2 - day 1 as a second level analysis of JP - JM ruled out general practice effects associated with performing the same tasks twice, as well as normal brain development that occurs in students over a two-month period. The right cerebellum activation observed in EPr - EM and EPi - EM for day 2, but not in JP - JM, is consistent with its transient role in practice-related learning (Raichle et al., 1994).

The English past tense debate has been a major issue in cognitive science, focusing on the role of grammatical rules and/or associative memories in acquiring knowledge of the past tense (Chomsky and Halle, 1968; Rumelhart and McClelland, 1986; Pinker, 1991; Marslen-Wilson and Tyler, 1997). One positron emission tomography (PET) study (Jaeger et al., 1996) reports activation of widespread cortical regions for past tense generation, but data have not been conclusive as to the regular/ irregular verb distinction. This is because task difficulty may account for the differences in brain activation. The present study contributes to this debate from a cross-linguistic point of view, comparing the identification of Japanese (L1) and English (L2) past tense verbs. We suggest that syntactic mechanisms, rather than domain-general associative mechanisms, may be crucial in identifying past tense verbs. There are two reasons for this suggestion. First, Japanese past tense forms basically follow several sets of morphosyntactical rules (Tsujimura, 1996). Acquiring English morphosyntax as L2 involves not only the explicit knowledge of placing '-ed' at the end of verbs, but also the knowledge of exceptional rules, such that some verbs do not end in '-ed'; an exception thus proves the rule even for irregular verbs. Secondly, neuroimaging studies have accumulated the evidence for the syntactic specialization of the left IFG (Stromswold et al., 1996; Embick et al., 2000; Friederici et al., 2000; Hashimoto and Sakai, 2002; Sakai et al., 2002), though more work is still needed for language development. The present study further demonstrates that appropriate L2 training guides brain function toward L1 specialization. Future studies with substantial numbers of monozygotic and dizygotic twins at various ages will clarify whether genetic or environmental factors are responsible for the behavioral and functional changes observed here. Our approach to evaluate educational methods in terms of not only indirect behavioral changes but direct functional changes takes a first step toward a new era in the brain science of education.

\section{Notes}

We thank Yoshiki Hotta for initial guidance in the twin studies; Masao Ito and Yasushi Miyashita for their encouragement and helpful suggestions; the twin study group of researchers at Graduate School of Medicine, University of Tokyo for their zygosity analyses; Toshio Mitsuhashi, Kazuyoshi Hirano, Masako Fukushima, Yasuo Tanabe, Akiko Katsumata and Yuko Sugiura for their help in the twin studies; Kei Suzuki, Satoshi Suo, Natsuki Saeki, Ryuichiro Hashimoto and Yoshinori Tatsuno for their technical assistance; and Minako Maki for administrative assistance. This study was supported by a Core Research for Evolutional Science and Technology (CREST) grant from the Japan Science and Technology Agency (JST) and by a Young Investigators' Grant from the Human Frontier Science Program (HFSP) to K.L.S.

Address correspondence to Kuniyoshi L. Sakai, Department of Cognitive and Behavioral Science, Graduate School of Arts and Sciences, University of Tokyo, Komaba, 3-8-1 Komaba, Meguro-ku, Tokyo 153-8902, Japan. E-mail: sakai@mind.c.u-tokyo.ac.jp.

\section{References}

Boysson-Bardies B (1999) How language comes to children: from birth to two years. Cambridge, MA: MIT Press.

Chee MWL, Tan EWL, Thiel T (1999) Mandarin and English single word processing studied with functional magnetic resonance imaging. J Neurosci 19:3050-3056.

Chomsky N, Halle M (1968) The sound pattern of English. Cambridge, MA: MIT Press.

Dehaene S, Dupoux E, Mehler J, Cohen L, Paulesu E, Perani D, Van de Moortele P-F, Lehéricy S, Le Bihan D (1997) Anatomical variability in the cortical representation of first and second language. Neuroreport 8:3809-3815.

Embick D, Marantz A, Miyashita Y, O'Neil W, Sakai KL (2000) A syntactic specialization for Broca's area. Proc Natl Acad Sci USA 97:6150-6154.

Epstein SD, Flynn S, Martohardjono G (1996) Second language acquisition: theoretical and experimental issues in contemporary research. Behav Brain Sci 19:677-714.

Friederici AD, Opitz B, von Cramon DY (2000) Segregating semantic and syntactic aspects of processing in the human brain: an fMRI investigation of different word types. Cereb Cortex 10:698-705.

Gopnik A, Meltzoff AN, Kuhl PK (1999) The scientist in the crib: mind, brains, and how children learn. New York: William Morrow.

Hashimoto R, Sakai KL (2002) Specialization in the left prefrontal cortex for sentence comprehension. Neuron 35:589-597.

Jaeger JJ, Lockwood AH, Kemmerer DL, Van Valin RD Jr, Murphy BW, Khalak HG (1996) A positron emission tomographic study of regular and irregular verb morphology in English. Language 72:451-497.

Johnson JS, Newport EL (1989) Critical period effects in second language learning: the influence of maturational state on the acquisition of English as a second language. Cognit Psychol 21:60-99.

Kim KHS, Relkin NR, Lee KM, Hirsch J (1997) Distinct cortical areas associated with native and second languages. Nature 388:171-174.

Klein D, Milner B, Zatorre RJ, Meyer E, Evans AC (1995) The neural substrates underlying word generation: a bilingual functionalimaging study. Proc Natl Acad Sci USA 92:2899-2903.

Lenneberg EH (1967) Biological foundations of language. New York: John Wiley.

Marslen-Wilson WD, Tyler LK (1997) Dissociating types of mental computation. Nature 387:592-594.

Pinker S (1991) Rules of language. Science 253:530-535.

Raichle ME, Fiez JA, Videen TO, Macleod AK, Pardo JV, Fox PT, Petersen SE (1994) Practice-related changes in human brain functional anatomy during nonmotor learning. Cereb Cortex 4:8-26.

Rumelhart DE, McClelland JL (1986) On learning the past tenses of English verbs. In: Parallel distributed processing-explorations in the microstructure of cognition, Vol. 2: psychological and biological models (McClelland JL, Rumelhart DE, PDP Research Group, eds), pp. 216-271. Cambridge, MA: MIT Press. 
Sakai KL, Noguchi Y, Takeuchi T, Watanabe E (2002) Selective priming of syntactic processing by event-related transcranial magnetic stimulation of Broca's area. Neuron 35:1177-1182.

Sakai KL, Homae F, Hashimoto R (2003) Sentence processing is uniquely human. Neurosci Res 46:273-279.

Stromswold K, Caplan D, Alpert N, Rauch S (1996) Localization of syntactic comprehension by positron emission tomography. Brain Lang 52:452-473.
Suzuki K, Sakai KL (2003) An event-related fMRI study of explicit syntactic processing of normal/anomalous sentences in contrast to implicit syntactic processing. Cereb Cortex 13:517-526.

Tsujimura N (1996) An introduction to Japanese linguistics. Cambridge, MA: Blackwell. 